\title{
Soluciones nutritivas para el germinado hidropónico de Hordeum vulgare
}

\author{
NUTRITIVE SOLUTIONS FOR THE HYDROPONIC GERMINATION OF Hordeum vulgate \\ Edson Ordoñez ${ }^{1}$, Enrique-III Idrogo ${ }^{2,3}$, Napoléon Corrales $^{1}$
}

\section{Resumen}

El objetivo del estudio fue determinar el efecto de diferentes dosis de soluciones nutritivas A y B en el agua de riego de germinado hidropónico (GH) de cebada (Hordeum vulgare) sobre el valor nutricional y rendimiento del germinado. Se trabajó con seis tratamientos: Control - T0: sin solución A y B; T1: $1.00 \mathrm{ml}$ A y $0.50 \mathrm{ml}$ B; T2: $0.50 \mathrm{ml}$ A y $0.125 \mathrm{ml} \mathrm{B}$; T3: $0.75 \mathrm{ml}$ Ay $0.25 \mathrm{ml} \mathrm{B}$; T4: $1.25 \mathrm{ml}$ Ay $0.75 \mathrm{ml} \mathrm{B}$; T5: $1.50 \mathrm{ml}$ Ay $1.00 \mathrm{ml} \mathrm{B}$, todos con seis repeticiones. Los resultados del análisis químico proximal y rendimiento del germinado fueron sometidos a un diseño completamente al azar. Las dosis de soluciones nutritivas influyeron significativamente en la totalidad de las variables evaluadas, siendo T3 quien demostró mejores valores de las variables evaluadas a excepción del contenido de cenizas, en tanto que T0, T4 y T5 presentaron los menores valores. Por lo tanto, se concluye que la combinación de soluciones nutritivas en dosis de $0.75 \mathrm{ml} \mathrm{A} \mathrm{y}$ $0.25 \mathrm{ml} \mathrm{B}$ es la más apropiada para obtener mejores resultados sobre el rendimiento y valor nutricional de $\mathrm{GH}$ de $H$. vulgare.

Palabras clave: cebada; hidroponía; producción; rendimiento; soluciones

\section{AbstraCt}

The objective of the study was to determine the effect of different doses of nutritive solutions A and B in the water of barley hydroponic fodder (Hordeum vulgare) on the nutritional value and germination performance. Six treatments were used: Control - T0: without solution A and B; T1: $1.00 \mathrm{ml} \mathrm{Aand} 0.50 \mathrm{ml} \mathrm{B}$ : T2: $0.50 \mathrm{ml}$ Aand $0.125 \mathrm{ml} \mathrm{B}$; T3: 0.75 $\mathrm{ml} \mathrm{A}$ and $0.25 \mathrm{ml} \mathrm{B}$; T4: $1.25 \mathrm{ml} \mathrm{A}$ and $0.75 \mathrm{ml} \mathrm{B}$; T5: $1.50 \mathrm{ml} \mathrm{A}$ and $1.00 \mathrm{ml} \mathrm{B}$, all with six repetitions per treatment. The results of the proximal chemical analysis and germination

${ }^{1}$ Centro de Investigación Pecuaria, Facultad de Ingeniería Zootecnia, Universidad Nacional Pedro Ruiz Gallo, Lambayeque, Perú

${ }^{2}$ Facultad de Ciencias Biológicas, Universidad Nacional Pedro Ruiz Gallo, Lambayeque, Perú

${ }^{3}$ E-mail: enrique.idrogo@cientificagroup.com

Recibido: 13 de julio de 2017

Aceptado para publicación: 12 de diciembre de 2017 
yield were subjected to a completely randomized design. The doses of nutritive solutions significantly affected all the evaluated variables, with T3 showing the best values of the evaluated variables except for the ash content, while T0, T4 and T5 showed the lowest values. Therefore, it is concluded that the combination of nutritive solutions in doses of $0.75 \mathrm{ml}$ A and $0.25 \mathrm{ml} \mathrm{B}$ is the most appropriate to obtain better results on the performance and nutritional value of barley hydroponic germination.

Key words: barley; hydroponics; production; solutions; yield

\section{INTRODUCCIÓN}

El término hidroponía deriva de dos palabras griegas: hydor, agua y ponos, trabajo, que combinadas significa «Trabajo en agua» y es una alusión al empleo de soluciones de agua y fertilizantes químicos para el cultivo de plantas sin tierra para su sustento (Miranda, 2006). El agua que se emplea tiene como condición básica de ser potabilizada, proveniente de pozo, lluvia o agua corriente de cañerías. En los casos que esta condición no se cumple, se requiere realizar un análisis químico. El pH del agua debe oscilar entre 5.2 y 7.0, salvo excepciones como en el uso de leguminosas, que pueden desarrollarse con un pH cercano a 7.5 (FAO, 2001).

El forraje hidropónico viene a ser el resultado del proceso de germinación de los granos de cereales o leguminosas (cebada, maíz, soya, sorgo) que se realiza durante 9 a 15 días, alcanzando una altura de 20 a $25 \mathrm{~cm}$ donde los animales consumen por completo los tallos, hojas, raíces y restos de semilla (Regalado, 2009). Se recomienda utilizar semillas de cereales libres de impurezas y que procedan de plantas sin enfermedades y que no hayan sido tratadas con fungicidas o preservantes. Las semillas deben estar enteras, secas y por lo menos con $85 \%$ de poder germinativo (Naik et al., 2015).

Con el uso de semilla de Hordeum vulgare (cebada) se espera un rendimiento de 6 a $8 \mathrm{~kg}$ de forraje hidropónico por kilo de semilla (Tarrillo, 2005) y una precocidad de germinación mayor al 90\% (Pérez-Ruiz et al.,
2015). H. vulgare es un alimento de alto rendimiento, con gran valor nutritivo que se puede producir durante todo el año (Cayllahua et al., 2015). Además, las enzimas se movilizan e invaden el interior de las semillas en el proceso de germinación, por lo que ocurre una disolución de paredes celulares por la acción de estas, liberándose granos de almidón que son transformados en azúcares (Fazaeli et al., 2012). Se han desarrollado tecnologías para producir biomasa vegetal utilizando soluciones nutritivas durante la etapa de germinación y el crecimiento temprano de la cebada, logrando un desarrollo entre 12 a 15 días, para su uso como forraje (Candia, 2014). El forraje verde hidropónico $(\mathrm{FVH})$ tiene como ventajas el ahorro de agua, eficiencia en el uso del espacio, eficiencia en el tiempo de producción, la mayor calidad del forraje para los animales y un menor costo de producción (FAO, 2001).

En la Región Lambayeque, Perú, la producción tradicional de germinado hidropónico (GH) busca optimizar la producción por kilogramo de semilla procesada utilizando agua pura para aprovechar el potencial germinativo de cada semilla; sin embargo, no se viene considerando la utilización de soluciones nutritivas para ayudar a potenciar el desarrollo productivo y calidad nutricional del GH producido. Trabajos previos demuestran la eficiencia del uso de soluciones hidropónicas en la producción de $\mathrm{GH}$ de $\mathrm{H}$. vulgare utilizando dosis recomendadas para hortalizas (Curay, 2013; Guevara, 2013; Ruesta, 2013), pero es poco lo que se conoce sobre dosis de soluciones nutritivas para optimizar la producción 
y valor nutricional del germinado hidropónico. El objetivo del estudio fue determinar la mejor dosis de una solución nutritiva en el agua de riego de GH de $H$. vulgare determinado a través de su valor nutricional y rendimiento.

\section{Materiales y Métodos}

\section{Lugar de Ejecución}

La fase de campo se realizó en el centro poblado Nuevo Mocse, Lambayeque, Perú, en diciembre de 2014 y los análisis de composición química se llevaron a cabo en el Laboratorio de Nutrición Animal de la Facultad de Ingeniería Zootecnia de la Universidad Nacional Pedro Ruiz Gallo, Lambayeque, Perú.

\section{Tratamientos}

La composición de la solución concentrada A fue de $550 \mathrm{~g}$ de nitrato de potasio, $350 \mathrm{~g}$ de nitrato de amonio y $180 \mathrm{~g}$ de superfosfato triple para un volumen final de 5 litros de agua, mientras que la solución B fue de $220 \mathrm{~g}$ de sulfato de magnesio, $17 \mathrm{~g}$ de quelato de hierro al $6 \% \mathrm{Fe} \mathrm{y} 400 \mathrm{ml}$ de solución de micronutrientes para un volumen final de 2 litros de agua.

Se trabajó con seis tratamientos utilizando agua de riego de $\mathrm{GH}$ de $H$. vulgare. T0: sin solución nutritiva; T1: $1.0 \mathrm{ml}$ de solución nutritiva A y $0.5 \mathrm{ml}$ de solución nutritiva $\mathrm{B}$ : T2: $0.50 \mathrm{ml}$ de solución nutritiva A y $0.125 \mathrm{ml}$ de solución nutritiva $\mathrm{B} ; \mathrm{T} 3: 0.75 \mathrm{ml}$ de solución nutritiva A y $0.25 \mathrm{ml}$ de solución nutritiva $\mathrm{B}$; T4: $1.25 \mathrm{ml}$ de solución nutritiva $\mathrm{A} y$ $0.75 \mathrm{ml}$ de solución nutritiva $\mathrm{B}$; T5: $1.5 \mathrm{ml}$ de solución nutritiva A y $1.0 \mathrm{ml}$ de solución nutritiva B. Las soluciones nutritivas fueron suministradas en el agua de riego desde el día 4 al 8 del proceso.

\section{Semilla de $H$. vulgare}

Se utilizó semilla de $H$. vulgare adquirida en el mercado mayorista Moshoqueque en la ciudad de Chiclayo. Se hizo una evaluación previa en tres locales comerciales para determinar el valor cultural $(76.8,84.0 \mathrm{y}$ $87.4 \%$ ), adquiriéndose $20 \mathrm{~kg}$ de semilla del local con mayor valor cultural.

Para instalar el sistema de cultivo hidropónico se emplearon 36 bandejas plásticas de $0.35 \mathrm{~m}$ x $0.42 \mathrm{~m}$, asignando seis bandejas por tratamiento. Se utilizó una densidad de siembra de $3 \mathrm{~kg} / \mathrm{m}^{2}$ recomendado por Guevara (2013), lo que implicó un total de $15.88 \mathrm{~kg}$ de semilla limpia ( $0.441 \mathrm{~g}$ de semilla por bandeja).

Se eliminaron los granos partidos, paja y otras impurezas presentes en la semilla adquirida, se lavó con agua para eliminar polvo y otras impurezas, y se desinfectó con hipoclorito de sodio al $0.001 \%$ durante dos horas. Luego se hizo un segundo lavado para eliminar el desinfectante, de modo de realizar el proceso de imbibición de las semillas por 24 horas. Al término de este procedimiento se colocaron las semillas en baldes de oreo debidamente tapados por un periodo de 48 horas.

\section{Germinación y Producción}

La etapa de germinación empezó después del oreo y cuando habían brotado las raíces. Se pesó el total de semilla y se dividió y sembró entre las 36 bandejas. Estas fueron trasladadas a las cámaras de germinación provista de una manta oscura donde permanecieron por cinco días. El riego se realizó cuatro veces al día (06:00, 12:00, 18:00, 24:00 horas) con ayuda de un aspersor manual. En el día 4 se inició el riego con las soluciones hidropónicas de las bandejas de los tratamientos T1 a T5, según la dosificación de cada uno. 
La etapa de producción se inicia el día 6 pos-siembra, donde se procedió a retirar la manta dejando al descubierto las bandejas por seis días más. En esta etapa se continuó el riego en $\mathrm{T} 1$ a $\mathrm{T} 5$ de cuatro veces al día con microaspersor hasta el día 8 pos-siembra y posteriormente regadas con agua pura hasta el día 15 , donde se realizó la cosecha del forraje hidropónico. Asimismo, en esta etapa se tomaron tres lecturas al día de temperatura y humedad relativa del ambiente (06:00, 12:00, 18:00 horas). Al final de la etapa de producción se registraron los pesos del forraje de cada bandeja.

\section{Variables Evaluadas}

Se determinó la producción de germinado hidropónico por metro cuadrado y la producción de materia seca de germinado hidropónico por metro cuadrado (TCO). Asimismo, la producción de proteína cruda $(\mathrm{PC})$, extracto etéreo (EE), fibra cruda (FC) y cenizas (CEN) de germinado hidropónico por metro cuadrado en base seca $(\mathrm{kg})$, rendimiento de germinado hidropónico fresco y de materia seca (MS) de germinado hidropónico por kilogramo de semilla procesada.

En el cálculo del rendimiento por metro cuadrado por tratamiento en base fresca se utilizó el área de la bandeja $\left(0.147 \mathrm{~m}^{2}\right)$ y los pesos en base fresca obtenidos a los 15 días de germinado hidropónico. Para calcular el aporte de MS se obtuvieron los datos de materia seca y la producción de germinado hidropónico en base fresca por metro cuadrado. Para calcular los aportes de PC, EE, FC y CEN se utilizó la información de aporte nutricional en base seca y la producción de MS.

E1 rendimiento de germinado hidropónico obtenido a partir de un kilogramo de semilla se calculó con base al peso por bandeja de cada tratamiento en base fresca. Asimismo, para obtener el rendimiento de $\mathrm{MS} / \mathrm{kg}$ de semilla procesada se utilizaron los niveles de MS las bandejas de cada tratamiento.

\section{Análisis Estadístico}

Se utilizó un diseño completamente al azar (DCA) con igual número de repeticiones por tratamiento ( 6 por tratamiento), cuyo modelo aditivo lineal según Padron (2009) es $\mathrm{Y}_{\mathrm{ij}}=\mu+\mathrm{t}_{\mathrm{i}}+\varepsilon_{\mathrm{ijk}}$, donde $\mathrm{Y}_{\mathrm{ij}}=$ Variable aleatoria observable correspondiente al i-ésimo tratamiento, y j-ésima repetición, $\mu=$ Media general, $\mathrm{t}_{\mathrm{i}}=$ Efecto del i-ésimo tratamiento, $\varepsilon_{\mathrm{ij}}=$ Error experimental que se presenta al efectuar la j-ésima observación del i-ésimo tratamiento, $\mathrm{t}=$ número de tratamientos, $\mathrm{r}=$ número de repeticiones

Se realizó el análisis de varianza para determinar diferencias estadísticas significativas entre los tratamientos $(p<0.05)$, y la prueba de comparación múltiple de Tuckey para identificar los tratamientos con significancia estadística.

\section{Resultados y Discusión}

La mejor producción de materia fresca de GH se obtuvo en T3 superando en 17.4\% a T0, tratamiento que presentó el menor rendimiento. No hubo diferencia estadística en producción de $\mathrm{MS} / \mathrm{m}^{2}$ de GH entre T0, T1 y T3; no obstante, la producción de T3 fue significativamente mayor que las observadas en T2, T4 y T5 ( $<<0.05$; Cuadro 1$)$.

La producción de PC fue similar en todos los tratamientos con excepción de T2, que mostró valores inferiores a T3 $(\mathrm{p}<0.05$; Cuadro 1). El valor de T3 fue mayor al obtenido por Quiñonez (2014) de $0.34 \mathrm{~kg}$, pero por debajo del nivel reportado por Curay (2013) de $0.52 \mathrm{~kg}$. Por otro lado, todos los valores de $\mathrm{PC} / \mathrm{m}^{2}$ fueron superiores al valor de $0.28 \mathrm{~kg} \mathrm{PC} / \mathrm{m}^{2}$ encontrado por Aguilar (2014).

La producción de EE fue similar en los tratamientos T0, T1 y T3. Por otro lado, los valores de T0 y T3 fueron superiores a los obtenidos en T2, T4 y T5 ( $<<0.05$; Cuadro 1). 
Cuadro 1. Producción $\left(\mathrm{kg} / \mathrm{m}^{2}\right)$ de materia fresca y seca, y proteína cruda, extracto etéreo, fibra cruda y cenizas en base seca, y rendimiento en base fresca (BF) y seca (MS) por kilogramo de semilla de germinado hidropónico de Hordeum vulgare (cebada)

\begin{tabular}{lcccccc}
\hline & $\mathrm{T} 0$ & $\mathrm{~T} 1$ & $\mathrm{~T} 2$ & $\mathrm{~T} 3$ & $\mathrm{~T} 4$ & $\mathrm{~T} 5$ \\
\hline Materia fresca & $20.42^{\mathrm{b}}$ & $22.48^{\mathrm{ab}}$ & $23.16^{\mathrm{ab}}$ & $23.97^{\mathrm{a}}$ & $22.40^{\mathrm{ab}}$ & $23.10^{\mathrm{ab}}$ \\
& \pm 1.47 & \pm 0.90 & 0.52 & \pm 2.99 & \pm 1.36 & \pm 1.75 \\
Materia seca (MS) & $2.62^{\mathrm{ab}}$ & $2.66^{\mathrm{ab}}$ & $2.46^{\mathrm{b}}$ & $2.82^{\mathrm{a}}$ & $2.32^{\mathrm{b}}$ & $2.58^{\mathrm{b}}$ \\
& \pm 0.19 & \pm 0.11 & \pm 0.06 & \pm 0.35 & \pm 0.14 & \pm 0.19 \\
Proteína cruda (PC) & $0.35^{\mathrm{ab}}$ & $0.38^{\mathrm{ab}}$ & $0.34^{\mathrm{b}}$ & $0.39^{\mathrm{a}}$ & $0.34^{\mathrm{ab}}$ & $0.38^{\mathrm{ab}}$ \\
& +0.02 & \pm 0.01 & \pm 0.01 & \pm 0.05 & \pm 0.02 & \pm 0.03 \\
Extracto etéreo (EE) & $0.11^{\mathrm{a}}$ & $0.10^{\mathrm{ab}}$ & $0.10^{\mathrm{b}}$ & $0.11^{\mathrm{a}}$ & $0.08^{\mathrm{c}}$ & $0.08^{\mathrm{c}}$ \\
& \pm 0.01 & \pm 0.01 & \pm 0.00 & \pm 0.01 & \pm 0.01 & \pm 0.01 \\
Fibra cruda (FC) & $0.31^{\mathrm{b}}$ & $0.32^{\mathrm{b}}$ & $0.33^{\mathrm{b}}$ & $0.41^{\mathrm{a}}$ & $0.26^{\mathrm{c}}$ & $0.33^{\mathrm{b}}$ \\
& \pm 0.02 & \pm 0.01 & \pm 0.01 & \pm 0.05 & \pm 0.02 & \pm 0.03 \\
Cenizas (CEN) & $0.07^{\mathrm{c}}$ & $0.14^{\mathrm{a}}$ & $0.12^{\mathrm{b}}$ & $0.12^{\mathrm{b}}$ & $0.08^{\mathrm{c}}$ & $0.04^{\mathrm{d}}$ \\
& \pm 0.01 & \pm 0.01 & \pm 0.00 & \pm 0.02 & \pm 0.01 & \pm 0.00 \\
Rendimiento base & $6.39^{\mathrm{b}}$ & $7.03^{\mathrm{ab}}$ & $7.24^{\mathrm{ab}}$ & $7.50^{\mathrm{a}}$ & $7.01^{\mathrm{ab}}$ & $7.23^{\mathrm{ab}}$ \\
fresca & \pm 0.46 & \pm 0.28 & \pm 0.16 & \pm 0.94 & \pm 0.42 & \pm 0.55 \\
& & & & & & \\
Rendimiento materia & $0.82^{\mathrm{ab}}$ & $0.83^{\mathrm{ab}}$ & $0.77^{\mathrm{b}}$ & $0.88^{\mathrm{a}}$ & $0.73^{\mathrm{b}}$ & $0.81^{\mathrm{ab}}$ \\
seca & \pm 0.06 & \pm 0.03 & \pm 0.02 & \pm 0.11 & \pm 0.04 & \pm 0.06 \\
\hline
\end{tabular}

Los valores obtenidos en el presente estudio fueron, no obstante, inferiores al valor de $0.13 \mathrm{~kg}$ de EE $/ \mathrm{m}^{2}$ hallado por Curay (2013), pero superiores a los valores reportados por Aguilar (2014) y Quiñonez (2014).

El mayor rendimiento de $\mathrm{FC}$ se obtuvo con el tratamiento T3 $\left(0.41 \mathrm{~kg} / \mathrm{m}^{2}\right)$ y el menor con $\mathrm{T} 4\left(0.261 \mathrm{~kg} / \mathrm{m}^{2}\right)($ Cuadro $1 ; \mathrm{p}<0.05)$. Este último valor fue mayor que los hallados por Aguilar (2014) y Quiñonez (2014). Por otro lado, la mayor producción de cenizas se presentó en $\mathrm{T} 1$ con $0.14 \mathrm{~kg} / \mathrm{m}^{2}$ de ceniza, seguido por T2 y T3 $(\mathrm{p}<0.05)$, ocurriendo la menor producción en T5 (p $<0.05$; Cuadro 1). Quiñonez (2014) y Aguilar (2014) obtuvieron 0.10 y $0.06 \mathrm{~kg} / \mathrm{m}^{2}$ sin utilizar soluciones hidropónicas en el agua de riego, y Curay (2013) reportó de $0.08 \mathrm{~kg} / \mathrm{m}^{2}$ utilizando estas soluciones en el agua de riego.
El rendimiento base fresca de GH por kilogramo de semilla fue estadísticamente similar en todos los tratamientos $(7.01-7.50 \mathrm{~kg})$ con excepción de T0 que fue inferior a T3 (6.39 kg; $p<0.05)$. Los valores de los tratamientos con soluciones nutritivas estuvieron dentro de los rangos reportados por otros autores que no utilizaron soluciones hidropónicas en el agua de riego (Tarrillo, 2005; Guevara, 2013; Quiñonez, 2014). Por otro lado, Aguilar (2014) y Ruesta (2013) reportaron rendimientos inferiores.

Por último, el rendimiento de materia seca por kilogramo de semilla procesada fue similar en T0, T1, T3 y T5 superando el rendimiento reportado por Lópezy Ruales (2010) de $0.62 \mathrm{~kg}$ y de Quiñonez (2014) con $0.78 \mathrm{~kg}$, quienes no utilizaron soluciones hidropónicas en el agua de riego. No obstante, fue bastan- 
te inferior al rendimiento de $1.7 \mathrm{~kg}$ en 17 días logrado por Sinchiguano (2008).

\section{Conclusiones}

El uso de soluciones hidropónicas en el agua de riego aumenta la producción y valor nutricional del germinado hidropónico de Hordeum vulgare, siendo la dosis más apropiada el tratamiento $\mathrm{T} 3 \mathrm{con} 0.75 \mathrm{ml}$ de solución concentrada A y $0.25 \mathrm{ml}$ de solución concentrada $\mathrm{B}$ diluidos en $4 \mathrm{~L}$ de agua de riego suministrados desde el día 4 hasta el día 8 de la siembra.

\section{Literatura Citada}

1. Aguilar M. 2014. Influencia del periodo de oscuridad en el rendimiento de germinado hidropónico de cebada (Hordeum vulgare) en Lambayeque. Tesis de Ingeniero Zootecnista. Lambayeque: Univ. Nacional Pedro Ruiz Gallo. 76 p.

2. Candia L. 2014. Evaluación de la calidad nutritiva de forraje verde de cebada Hordeum vulgare hidropónico, fertilizado con soluciones de guano de cuy $\mathrm{Ca}$ via porcellus a dos concentraciones. Salud Tecnol Vet 2: 55-62. doi: 10.20453/ stv.v2i1.2202

3. Cayllahua CF, Condori DD, Cordero FA, Veliz LM, Contreras PJ. 2015. Sustitución gradual de la alfalfa (Medicago sativa 1.) por el germinado de cebada (Hordeum vulgare) en raciones de cuyes (Cavia porcellus L) en la etapa de crecimiento. Rev Complutense Cienc Vet 9: 7-21. doi: 10.5209/rev_RCCV.2015.v9.n2.49601

4. Curay I. 2013. Cultivo hidropónico de cebada (Hordeum vulgare) con y sin solución hidropónica en el agua de riego. Tesis de Ingeniero Zootecnista. Lambayeque: Univ. Nacional Pedro Ruiz Gallo. 67 p.
5. Fazaeli H, Golmohammadi HA, Tabatabayee SN, Asghari-Tabrizi M. 2012. Productivity and nutritive value of barley green fodder yield in hydroponic system. World Appl Sci J 16: 531-539.

6. [FAO] Food and Agriculture Organization of the United Nations. 2001. Forraje verde hidropónico. Santiago de Chile: Oficina Regional de la FAO para América Latina y el Caribe. 68 p.

7. Guevara S. 2013. Rendimiento de germinado hidropónico (G.H.) de cebada (Hordeum vulgare $\mathrm{L}$ ) en seis niveles de densidad de siembra. Tesis de Ingeniero Zootecnista. Lambayeque: Univ. Nacional Pedro Ruiz Gallo. 67 p.

8. Lopéz D, Ruales J. 2007. Evaluación de edad de cosecha y niveles de forraje verde hidropónico de cebada, maíz y trigo en el crecimiento de conejos de carne (Oryctolagus cuniculus) raza Neozelandés. Tesis de Ingeniero Agropecuario. Ibarra: Univ. Técnica del Norte. $18 \mathrm{p}$.

9. Miranda I. 2006. Fertilizantes foliares en cultivo hidropónico de cebada (Hordeum vulgare). Tesis de Ingeniero Zootecnista. Lambayeque: Univ. Nacional Pedro Ruiz Gallo. 49 p.

10. Naik PK, Swain BK Cordero FA, Singh NP. 2015. Production and utilisation of hydroponics fodder. Indian J Anim Nutrit 32: 1-9.

11. Padron E. 2009. Diseños experimentales con aplicación a la agricultura y ganadería. México: Ed Trillas. $224 \mathrm{p}$.

12. Pérez-Ruiz J, Mejia-Contreras J, Hernández-Livera A, Zamora-Díaz $M .2015$. Ausencia de latencia en semillas de genotipos mexicanos de cebada (Hordeum vulgare L) para malta. Rev Fitotec Mex 38: 249-255.

13. Quiñonez P. 2014. Influencia del ciclo lunar en la producción de germinado hidropónico de cebada (Hordeum vulgare) en Lambayeque. Tesis de Ingeniero Zootecnista. Lambayeque: Univ. Nacional Pedro Ruiz Gallo. 56 p. 
14. Regalado F. 2009. Cultivos hidropónicos. Lambayeque: Univ. Nacional Pedro Ruiz Gallo. 48 p.

15. Ruesta I. 2013. Tiempo de remojo y concentración de yodo y/o lejía en desinfección de semilla de germinado hidropónico de cebada (Hordeum vulgare $\mathrm{L}$ ) en Lambayeque. Tesis de Ingeniero Zootecnista. Lambayeque: Univ. Nacional Pedro Ruiz Gallo. 105 p.
16. Sinchiguano M. 2008. Producción de forraje verde hidropónico de diferentes cereales (avena, cebada, maíz, trigo y vicia) y su efecto en la alimentación de cuyes. Tesis de Ingeniero Zootecnista. Riobamba: Escuela Politécnica de Chimborazo. $108 \mathrm{p}$.

17. Tarrillo H. 2005. Forraje verde hidropónico. Manual de producción. Lima: Centro de Investigación de Hidroponía y Nutrición Mineral, Univ. Nacional Agraria La Molina. 41 p. 